International Conference on Renewable Energies and Power Quality (ICREPQ'13)
Bilbao (Spain), 20 $20^{\text {th }}$ to $22^{\text {th }}$ March, 2013
Renewable CEnergy and POaver Qualily. Yournal (RE\&PQJ)
ISSN 2172-038 X, No.11, March 2013

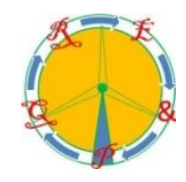

\title{
On-line estimation of induction generator parameters using adaptive neuro-fuzzy inference systems for wind energy conversion systems
}

\author{
A. Mesemanolis, and C. Mademlis \\ Department of Electrical and Computer Engineering \\ Aristotle University of Thessaloniki \\ Thessaloniki, 54124, Greece \\ Phone/Fax: 00302310996326 , \\ E-mail: athmesem@auth.gr, mademlis@eng.auth.gr
}

\begin{abstract}
This paper proposes a new method for online estimation of the induction generator parameters by means of adaptive neuro-fuzzy inference systems (ANFIS). The suggested technique can be applied to induction generators that are used in wind energy conversion systems (WECS). The WECS structure comprises a wind turbine, a three-phase induction generator and two back-to-back power converters. The WECS provides electric energy to the utility grid through an LCL filter. The selfadjustment of the induction generator parameters provides accuracy in the implementation of the field oriented control and therefore accomplishes optimal operation on the WECS. The proposed method is simple and, since it does not require time consuming off-line laboratory experiments, it can be easily applied to any wind energy system that is already in operation. Several simulation results will be presented in order to validate the theoretical considerations and demonstrate the operational improvements of the proposed system.
\end{abstract}

\section{Key words}

Induction generator, wind energy conversion system, variable speed Drives, adaptive neuro-fuzzy systems

\section{Introduction}

Wind energy has attracted increased attention during the past years, as they are considered as viable solution to the world's ever growing power needs. Squirrel cage rotor induction generator is an attractive option for a wind generation system, since it is inexpensive with robust construction compared to permanent magnet synchronous generator and also requires low maintenance compared to wound rotor synchronous generator. Variable speed WECSs have been widely investigated in the technical literature and several developments in the generator control have been proposed.

Field oriented control is widely applied in WECS with induction generator because it provides decoupled control of torque and flux-linkage. Thus, fast tracking of the desired speed and independent regulation of the fluxlinkage can be achieved. In order to achieve decoupled control, the torque and flux components of the stator current are aligned with the $q$ and $d$ axes, respectively. Critical parameters for the implementation of the field oriented control are the rotor resistance and inductance, which can be considered constant or can be estimated online by various methods.

For ideal decoupled control, the controller parameters should track the respective machine parameters. However, this is quite difficult to be achieved because rotor resistance varies with temperature and rotor inductance depends on the machine's saturation level.

Several research papers have been published in the technical literature for on-line identification of induction machine parameters [2]-[11]. However, none of them takes into account the variation of the magnetizing inductance that may cause slip miscalculation and consequently sluggish flux-loop response. This would also affect the torque sensitivity and consequently would lengthen the response time of the whole WECS control. In [15] and [16], a control strategy for varying parameter and saturated machines are presented, respectively; however, the machine parameters are calculated off line and then remain constant. In [1], an online identification method for the magnetization curve of an induction motor is presented; however, it relies on measurement of the stator voltage, which is not always possible due to hardware limitations. Furthermore, the calculation of the magnetization inductance may fail, because the method assumes alignment of the $d-q$ transformation with the stator flux vector, which is dependent on the magnetization inductance and therefore is uncertain.

In this paper, an on-line identification method for the magnetization curve of a squirrel cage induction generator is proposed. The suggested method is applied on a variable speed WECS, but it can also be applied on a general purpose variable speed squirrel cage induction 
machine driven by a power converter and a variable speed control system. The proposed method is simple and can be applied on-line; therefore, it does not require time consuming and costly off line measurements.

The identification of the machine magnetization curve is performed by considering the induction machine differential equations, expressed on the synchronous $d-q$ reference frame. The magnetization curve is initially considered linear and a training algorithm on-line finetunes it, by considering the electric loss of the induction generator and the power output of the system. Specifically, the measured power output of the generator is compared with the estimated power output and through this comparison, the instantaneous value of the magnetization inductance for a given set values of $d$ - and $q$ - axis current components is estimated.

In order to approximate the magnetization inductance of the machine as a function of the machine currents, usually a piecewise linear or a polynomial function of the magnetizing current is used [1]. In this paper, a non-linear adaptive Neuro-Fuzzy inference system (ANFIS) is used for the approximation of the magnetization curve. The advantage of such choice is the ability of Neuro-Fuzzy systems to approximate any kind of non-linear curve with increased accuracy and a minimal number of adaptive neurons. This means that the computational effort needed is relatively small compared to the increased precision of the approximation, and a minimal number of parameters is needed for the tuning of the system. Furthermore, the modern microprocessors are powerful enough to perform such calculations in real time. The ANFIS approximation provides filtering of the input signals through the fuzzification process, reducing the noise of the system that is caused by the PWM technique of variable speed inverters.

\section{Induction machine model}

The equations of an induction machine on the synchronously rotating $d q$ - reference frame are

$$
\left[\begin{array}{c}
\frac{d i_{s q}}{d t} \\
\frac{d i_{s d}}{d t} \\
\frac{d i_{r q}}{d t} \\
\frac{d i_{r d}}{d t}
\end{array}\right]=A\left[\begin{array}{l}
i_{s q} \\
i_{s d} \\
i_{r q} \\
i_{r d}
\end{array}\right]+B\left[\begin{array}{l}
u_{s q} \\
u_{s d}
\end{array}\right]
$$

with

$$
\begin{aligned}
A & =\frac{1}{\sigma}\left[\begin{array}{cccc}
L_{r} R_{s}-L_{m} R_{r}^{\prime} & L_{m}^{2} \omega_{r}-\sigma \omega_{e} & 0 & L_{m} L_{r} \omega_{r} \\
-L_{m}^{2} \omega_{r}+\omega_{e} & L_{r} R_{s}-L_{m} R_{r}^{\prime} & -L_{m} L_{r} \omega_{r} & 0 \\
L_{s} R_{r}^{\prime}-L_{m} R_{s} & -L_{m} L_{s} \omega_{r} & 0 & -L_{r} L_{s} \omega_{r}-\sigma \omega_{e} \\
L_{m} L_{s} \omega_{r} & L_{s} R_{r}^{\prime}-L_{m} R_{s} & L_{r} L_{s} \omega_{r}+\sigma \omega_{e} & 0
\end{array}\right] \\
B & =\frac{1}{\sigma}\left[\begin{array}{cc}
-L_{r} & 0 \\
0 & -L_{r} \\
L_{m} & 0 \\
0 & L_{m}
\end{array}\right]
\end{aligned}
$$

where $\sigma=L_{m}^{2}-L_{r} L_{s}$. The electromagnetic torque of the machine is given by

$$
T_{e}=\frac{3}{2} p L_{m}\left(i_{s q} i_{r d}-i_{s d} i_{r q}\right)
$$

The above expression of the machine equations is valid for any synchronously rotating reference frame; therefore, the following procedure is independent from the knowledge of the machine inductance.

In the above equations, the machine parameters can be easily determined from the no-load and locked rotor test of the electrical machine. However, the magnetization inductance of the generator, $L_{m}$, may be miscalculated or even change during the operation of the generator due to the effects of core saturation.

From (2), it is deduced that the calculation of the electromagnetic torque will produce false results when the magnetization inductance of the machine is unknown. The same is valid for the electromagnetic power at the shaft calculated as $P_{e}=T_{e} \omega_{r}$. However, when the machine operates with a back-to-back converter, the power output is measured either at the intermediate dclink or at the inverter output as a result of the system control demands. Thus, the real power output of the system is known. The error between the measured and the calculated machine power at the output is calculated as

$$
e_{p}=P_{\text {out }}-P_{m, \text { calc }}=P_{\text {out }}-\left(P_{e}-P_{c u}-P_{f e}\right)
$$

Due to power balance, the actual error cannot be other than zero and thus eq. (3) becomes:

$$
P_{\text {out }}=P_{e}-P_{c u}-P_{f e}=T_{e} \omega_{r}-i_{s}^{2} R_{s}-i_{r}^{2} R_{r}-i_{m}^{2} R_{f e}
$$

From (4), the electromagnetic torque of the generator can be calculated.

Considering a steady state of the machine, the left side of equation (1) is zero. For the field oriented control the stator currents are measured and the stator voltage command signals are calculated as a result of the control.

The rotor currents can be calculated solving (1) as:

$$
\begin{aligned}
& i_{r d}=\frac{-B_{11} u_{s q}-A_{11} i_{s q}-A_{12} i_{s d}}{A_{14}} \\
& i_{r q}=\frac{-B_{11} u_{s q}+A_{12} i_{s q}-A_{11} i_{s d}}{A_{14}}
\end{aligned}
$$

where $A_{11}, A_{12}, A_{14}, B_{11}$ are the corresponding elements of the matrices $A$ and $B$, respectively.

Substituting (5) into (2) we get: 


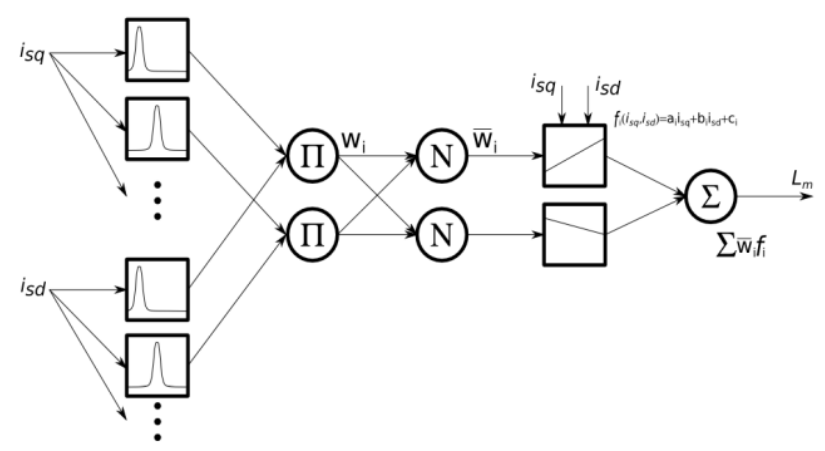

Fig. 1. Structure of the ANFIS for the approximation of the magnetization inductance

$$
T_{e}=\frac{3}{2} \frac{p L_{m}\left[\left(L_{r} R_{s}-L_{m} R_{r}^{\prime}\right) A-2\left(L_{m}^{2} \omega_{r}\right) B-L_{r} C\right]}{L_{m} L_{r} \omega_{r}}
$$

with $A=i_{s d}^{2}-i_{s q}^{2}, B=i_{s q} i_{s d}, C=u_{s d} i_{s d}-u_{s q} i_{s q}$.

Solving for $L_{m}$ and considering that $L_{r}=L_{m}+L_{l r}$, $L_{s}=L_{m}+L_{l s}$, a second order equation is produced and the solution can be calculated as:

$$
L_{m}=\frac{-\beta+\sqrt{\beta^{2}-4 \alpha \gamma}}{2 \alpha}
$$

with

$$
\begin{aligned}
& \alpha=-2 \omega_{r} B \\
& \beta=-\frac{2}{3} \frac{T_{e}}{p} \omega_{r}+\left(R_{s}-R_{r}\right) A-2\left(L_{l s}+L_{l r}\right) \omega_{e} B-C \\
& \gamma=-\frac{2}{3} \frac{T_{e}}{p} L_{l s} \omega_{r}+L_{l r} R_{s} A-2 L_{l s} L_{l r} \omega_{e} B-L_{l r} C
\end{aligned}
$$

In (7), only the positive root of the discriminant is calculated, as the negative yields negative solution for the magnetization inductance.

The above equations can be used to determine the magnetization inductance of the induction generator online, during the operation of the generator. However, the calculations are prone to numerical instability caused by the signal noise and the fluctuation of the generator currents and voltages during transients. Therefore, the results of the on-line estimation of the magnetization inductance cannot be trusted and they must be further processed in order to produce reliable results.

In order to gain a reliable approximation of the magnetization inductance, the data are gathered and used to train an ANFIS that will serve as an approximator of the magnetization curve. The ANFIS training can provide the necessary filtering of the acquired data.

The magnetization inductance of the generator is a function of the direct and quadrature axis current of the

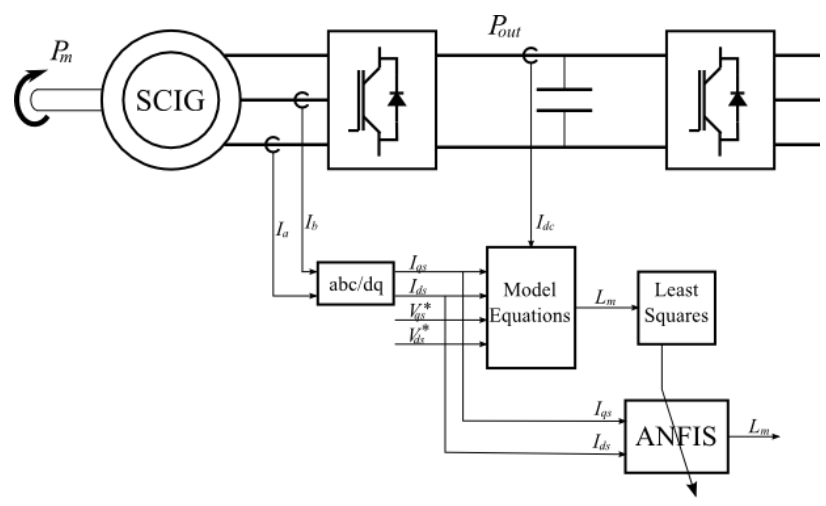

Fig. 2. Training procedure of the ANFIS. The magnetization inductance value is calculated through the model equations and the least squares algorithm trains the ANFIS with the new value of $L_{m}$.

generator. Thus, input-output pairs are stored and the ANFIS is trained, either on-line, or off-line, depending on the needs of the application. After the training is finished, the ANFIS can be used for the calculation of the magnetization inductance as a function of the machine currents.

\section{ANFIS structure}

The structure of the ANFIS that approximates the magnetization curve is given in Fig. 1. The ANFIS has the direct and quadrature axis current components of the stator current as inputs and the output is the magnetization inductance.

At the input layer, the two currents are sampled and fuzzified according to pre-decided fuzzy rules. The membership function of each rule pair for the two currents is decided through bell shaped membership functions, linguistically expressed as:

$$
\text { "if } i_{s q} \text { is } A_{i} \text { and } i_{s d} \text { is } B_{i} \text {, then the } L_{m} \text { is } f_{i} \text { " }
$$

The subscript $i$ corresponds to the $\mathrm{i}$-th fuzzy rule pair, and $A$ and $B$ are the fuzzy sets $\{$ small, medium, large $\}$. The function that corresponds to each fuzzy rules pair is calculated as

$$
f_{i}\left(i_{s q}, i_{s d}\right)=a_{i} i_{s q}+b_{i} i_{s d}+c_{i}
$$

The firing strength of each rule depends on the weight of each membership function. The output function corresponding to each rules pair is accordingly weighted with the firing strength of each rule pair, and the output of the ANFIS is calculated as

$$
\frac{\sum_{i}^{n} w_{i} f_{i}\left(i_{s q}, i_{s d}\right)}{\sum_{i}^{n} w_{i}}
$$

The training of the system is performed with linear recursive least squares identification of the ANFIS 


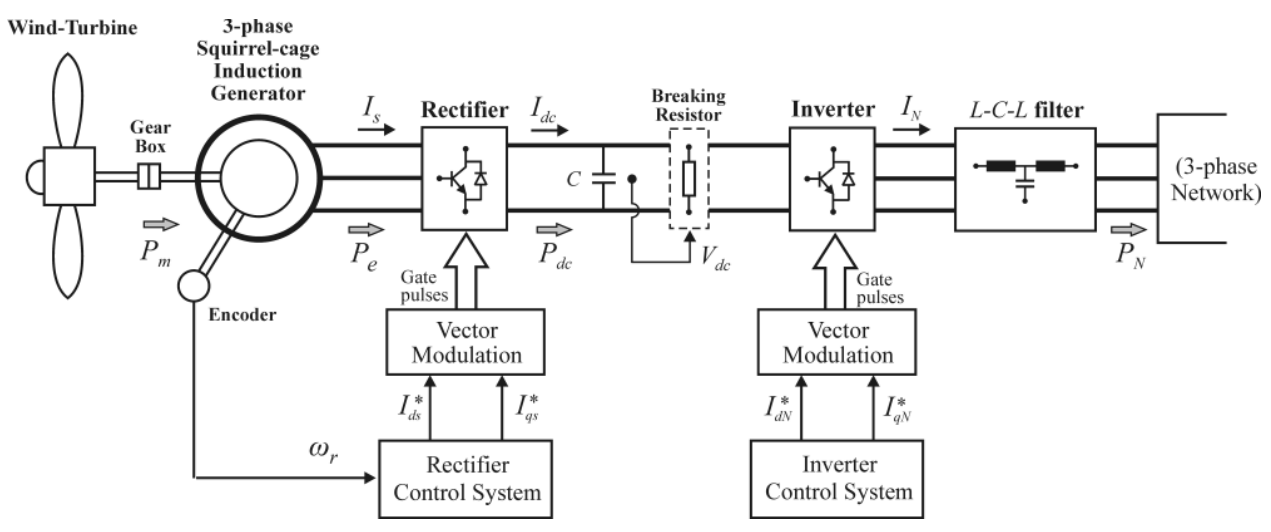

Fig. 3. System structure of a variable speed WECS with 3-phase squirrel-cage induction generator.

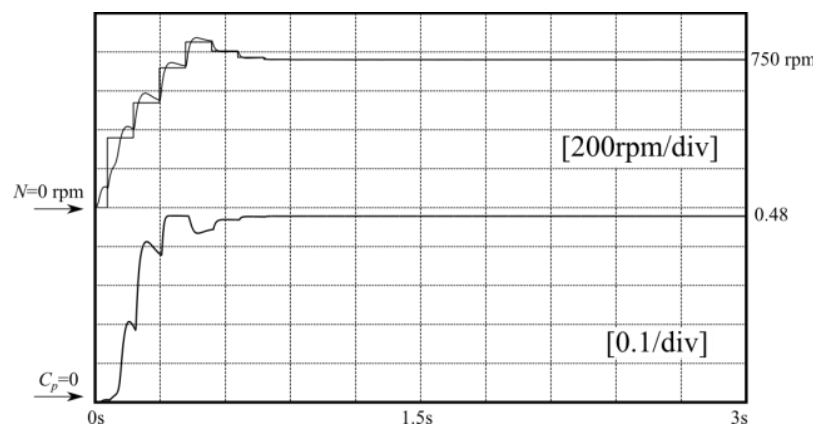

Fig. 4. Reference and real rotational speed of the generator and power coefficient of the WECS. The operation of the maximum power point tracker is demonstrated, and the system converges at $C_{p}=0.48$ which is the optimal value. The speed values refer to the generator speed.

parameters, as illustrated in Fig. 2. Initially, a linear magnetization curve is considered and the ANFIS is trained accordingly. At each identification step, the magnetization inductance of the generator is calculated with respect to the values of $i_{s q}$ and $i_{s d}$. The ANFIS is then trained in order to include the new data and the coefficients $a_{i}, b_{i}, c_{i}$ are adapted to the new value.

The convergence of the algorithm can be adjusted by choosing appropriate values for the initial covariance matrix $P_{k}$ that is required by the recursive least squares algorithm. A forgetting factor should also be chosen between $0.95-1$ so that the new values will gain more weight during the training.

For the application of the technique, initially an $L_{m}$ value is calculated through the no-load test of the machine. Then, the ANFIS is trained with the constant value for all $d$-axis currents and the system operates as normally. During the operation of the generator, the approximation of the magnetization curve constantly converges to its real value. After the training has been completed on various operating points of the generator, the magnetization curve can be retrieved and applied to the generator control scheme.

\section{Simulation Results}

A number of simulations have been conducted in order to demonstrate the operational improvements of the proposed identification method and the results are presented. For the simulation, a $5.5 \mathrm{~kW}$ Wind Energy Conversion System has

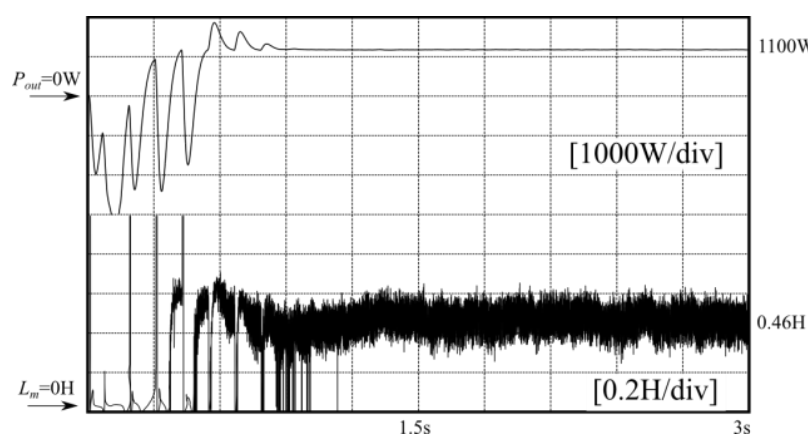

Fig. 5 Power output of the WECS and estimated value of the magnetization inductance, as calculated by the proposed method. The estimated inductance converges to its real value as the system approaches steady state.

been considered, as illustrated in Fig. 3. The system comprises a wind turbine coupled to a squirrel cage induction generator through a gear box, which is needed to adapt the low rotational speed of the wind turbine to that of the generator, which is typically three or four times higher. A power converter is used, that controls the speed of the generator and acts as a rectifier, transforming the alternating current that is produced by the generator to $\mathrm{dc}$ and injecting it to the dc-link. A capacitor is used at the dc-link to stabilize the dc voltage and a breaking resistor is installed in order to dissipate the excess electrical power that cannot be absorbed by the grid. Thus, the system is protected from overvoltage at the dc-link, for example during an abrupt wind gust or during a grid side fault, where the system cannot provide the generated power to the grid.

An inverter is placed at the output of the system, transforming the direct current to alternating with the appropriate frequency and phase and injecting it into the utility grid. An LCL filter is placed between the inverter and the utility grid that reduces the harmonic content of the injected current, according to the demands of the grid manager.

For the simulation, a wind turbine with blade radius of $2.25 \mathrm{~m}$ is considered and the gearbox gear ratio is chosen 1:4. The generator side converter (rectifier) utilizes vector control in order to control the speed and torque of the generator. Using rotor field oriented control (FOC), the $d$-axis current of the generator is aligned to the magnetic flux vector of the generator, so as the $q$-axis 


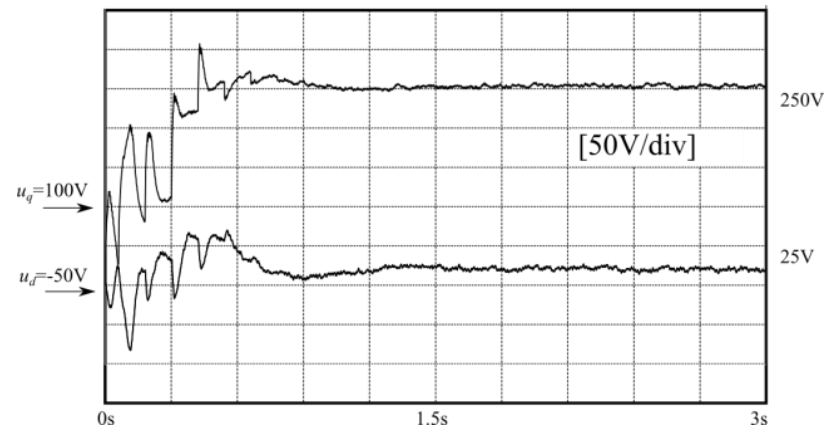

Fig. 6. $d$ - and $q$ - axis reference voltages of the generator stator as generated at the output of the current control loop proportional-integral controllers.

current is directly proportional to the generator torque and the $d$-axis current regulates the flux-linkage.

For the line side converter, vector control is also utilized, with the direct axis of the $d q$-transformation aligned with one of the line voltages, so as the $d$-axis controls the active power injection at the grid, while the $q$-axis current controls the reactive power. The power factor at the system output can be leading, lagging or unity, depending on the grid manager demands and can be adjusted during operation of the system. The LCL filter is chosen $4.2 \mathrm{mH}$ at for each inductance and a capacitor of $5 \mu \mathrm{F}$ is chosen. For the dc-link, a capacitor of $3 \mathrm{mF}$ is used and the breaking resistor is $50 \Omega$.

For the demonstration of the operation of the algorithm, a simulation has been conducted showing the calculation of the value of $L_{m}$. The system is operating with a constant wind of $6.5 \mathrm{~m} / \mathrm{s}$ and an MPPT algorithm is utilized in order to track the rotational speed of the wind turbine that achieves maximum power production, according to the wind turbine characteristic curves. The chosen MPPT algorithm is that of perturbation and observation which introduces a perturbation at the generator operating speed and measures the power output of the system. Following, the operating speed command signal is decided towards the speed that produced more power, and the algorithm keeps adding perturbations to the speed until equilibrium is reached, where the power production will be maximum and therefore MPP is achieved.

In Figs. 4 through 7, the simulation results for operation of the WECS with steady wind of $6.5 \mathrm{~m} / \mathrm{s}$ are presented. The system starts from zero speed and operates with MPPT algorithm, reaching the optimal speed within 1 second and then maintains steady state. The speed values refer to the generator side of the gearbox.

For the implementation of the FOC of the generator, a value for the magnetization inductance, different from the machine inductance has been chosen. The value of $L_{m}$ is used for the calculation of the flux-linkage of the machine; therefore, having a false value the FOC fails to align the $d$ axis of the $d q$ - transformation with the flux-linkage and the magnetic flux and torque of the generator are distributed unevenly. The machine model $L_{m}$ is $0.46 \mathrm{H}$ and the model is not saturated, which means that the $L_{m}$ is constant, while the FOC operates having a different value, at $0.6 \mathrm{H}$. Due to the closed loop operation of the generator, the speed of the

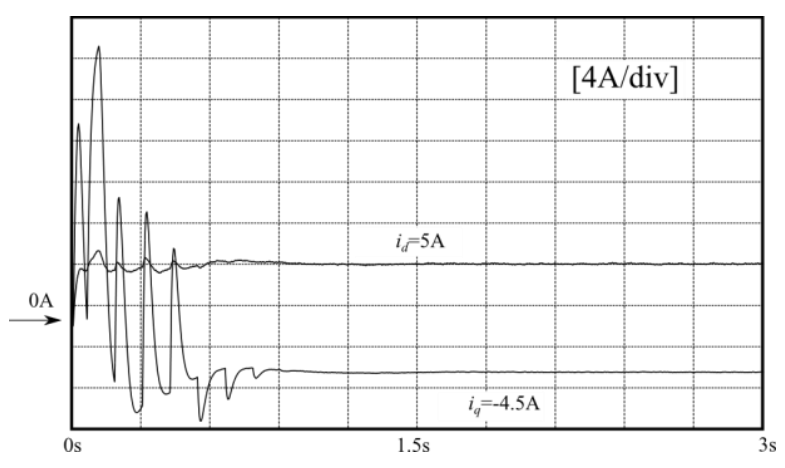

Fig. 7. $d$ - and $q$ - axis stator currents of the generator. The initial spikes of $i_{q}$ is due to the effect of the proportional-integral controller of the speed control loop and are reduced as the system reaches steady state. The negative value of $i_{q}$ is due to the operation as generator.

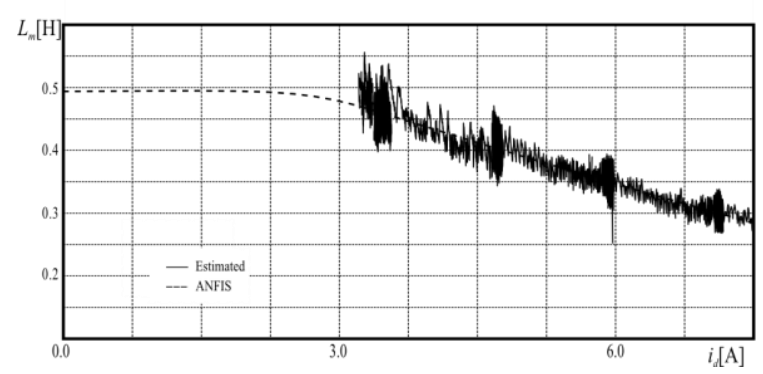

Fig. 8. Estimated and ANFIS-fitted value of the magnetization inductance $L_{m}$ of the generator.

generator will converge to the command speed, as calculated by the MPPT algorithm, however the response will be suboptimal, leading to possibly saturation of the machine or even instability and control failure.

The operation of the MPPT algorithm is demonstrated, as the system starts at an initial speed and then searches for the maximum power point, which is achieved at $0.8 \mathrm{~s}$. This can be confirmed by the $C_{p}$ coefficient, which reaches the optimum of 0.48 , meaning that $48 \%$ of the available wind speed is captured by the wind turbine. After the optimum has been reached, the generator achieves steady state and operates with constant speed.

As illustrated in Fig. 5, the algorithm output of the magnetization inductance estimation converges to the real value after the system has reached a steady state. This is because the estimation produces correct values only in steady state. Due to the noise that affects the signals fed to the algorithm, the output is also noisy and it needs to be filtered through the ANFIS.

Next, the ability of the ANFIS to approximate the magnetization curve is demonstrated. In order to simulate the entire magnetization curve, a saturated machine has been used and a series of simulations have been conducted with the generator operating at different levels of magnetization. In simulation, the effect of the $q$ - axis current component of the stator does not affect the magnetization level of the machine; therefore, it has not been regarded, and only the effect of $i_{d}$ has been taken into consideration. The data needed for the training of the ANFIS are the $d$ - axis current component of the generator and the corresponding estimated values of $L_{m}$. The 
ANFIS is initially trained with a constant value of $L_{m}=$ $0.47 \mathrm{H}$ for any value of the $d$-axis current component. Then, the ANFIS is trained on-line with data gathered during the simulation. The magnetization inductance versus $d$-axis current is illustrated in Fig. 8, along with the final form of the ANFIS, after the training. It can be seen that the ANFIS approximates successfully the magnetization inductance of the generator.

In the range 0-3.0 A the induced flux linkage at the rotor is too low; as a result the generator cannot operate in these conditions. Therefore, there have not been data for this area. However, the magnetization level at this region is low and the magnetization inductance is assumed constant, at the nominal value. The ANFIS training procedure does not alter the form of the curve at this region, since no training data have been provided.

After the training, the ANFIS can be extracted and used as reference for the $L_{m}$ value, with the input of the $d$-axis current component. The data is expected to also fit experimental results and the $q$ - axis current component effect on the magnetization can be observed as well.

\section{Conclusions}

In this paper, an on-line estimation method for the magnetization curve of induction machines is presented. The proposed method can operate on any general purpose variable speed squirrel cage induction machine driven by a power converter and a variable speed control system. The magnetization inductance is calculated in real time by means of the machine equations and an adaptive NeuroFuzzy inference system is trained to approximate the magnetization inductance as a function of the machine stator current. After the training of the ANFIS has been completed, the magnetization curve can be obtained and used for the improvement of the control of the machine. Several simulation results have been presented in the full paper in order to validate the operational improvements of the proposed algorithm.

\section{Acknowledgement}

This work was supported by European Regional Development Funds and Greek National Resources (Ministry of Education, Lifelong Learning Fund and Religious Affairs, Greece under Research Grant 09SYN32-624 (Research Program "Cooperation 2009") and the Research Committee of the Aristotle University of Thessaloniki.

\section{References}

[1] D. Chatterjee, "A Novel Magnetizing-Curve Identification and Computer Storage Technique for Induction Machines Suitable for
Online Application," Industrial Electronics, IEEE Transactions on , vol.58, no.12, pp.5336-5343, Dec. 2011

[2] A. Mesemanolis, C. Mademlis and I. Kioskeridis, "HighEfficiency Control for a Wind Energy Conversion System With Induction Generator," Energy Conversion, IEEE Transactions on , vol.PP, no.99, pp.1-10, in press

[3] M. Nasir Uddin and S. W. Nam, "New online loss-minimizationbased control of an induction motor drive," IEEE Trans. Power Electron., vol. 23, no. 2, pp. 926-933, Mar. 2008

[4] F. Corcoles, J. Pedra, M. Salichs and L.Sainz, "Analysis of the induction machine parameter identification," Energy Conversion, IEEE Transactions on , vol.17, no.2, pp.183-190, Jun 2002.

[5] K. Kampisios, P. Zanchetta, C. Gerada and A. Trentin, "Identification of Induction Machine Electrical Parameters Using Genetic Algorithms Optimization," Industry Applications Society Annual Meeting, 2008. IAS '08. IEEE, vol., no., pp.1-7, 5-9 Oct. 2008

[6] J. Cortes-Romero, C. Garcia-Rodriguez, A. Luviano-Juarez and H. Sira-Ramirez, "Algebraic parameter identification for induction motors," IECON 2011 - 37th Annual Conference on IEEE Industrial Electronics Society, vol., no., pp.1734-1740, 7-10 Nov. 2011

[7] B. Karanayil, M. F. Rahman, C. Grantham; , "Online Stator and Rotor Resistance Estimation Scheme Using Adaptive Neural Networks for Vector Controlled Speed Sensorless Induction Motor Drive," Industrial Electronics, IEEE Transactions on , vol.54, no.1, pp.167-176, Feb. 2007

[8] K. Wang, J. Chiasson, M. Bodson and L. M. Tolbert, "An Online Rotor Time Constant Estimator for the Induction Machine," Control Systems Technology, IEEE Transactions on , vol.15, no.2, pp.339-348, March 2007

[9] D. Telford, M. Dunnigan, B.W. Williams, "Online identification of induction machine electrical parameters for vector control loop tuning," Industrial Electronics, IEEE Transactions on , vol.50, no.2, pp. 253- 261, Apr 2003

[10] A. do Prado, A.H. de Sousa, S.M. Ferrari, "On-Line Identification of Induction Motors using Discrete Models for Sinusoidal Signals," IEEE Industrial Electronics, IECON 2006 - 32nd Annual Conference on , vol., no., pp.811-816, 6-10 Nov. 2006

[11] A. Oteafy, J. Chiasson and M. Bodson, "Online identification of the rotor time constant of an induction machine," American Control Conference, 2009. ACC '09. , vol., no., pp.4373-4378, 1012 June 2009

[12] M.L. Campbell, J. Chiasson, M. Bodson, L.M. Tolbert, "Speed Sensorless Identification of the Rotor Time Constant in Induction Machines," Automatic Control, IEEE Transactions on , vol.52, no.4, pp.758-763, April 2007

[13] Y. He, Y. Wang, Y. Feng and Z. Wang; , "Parameter Identification of an Induction Machine at Standstill Using the Vector Constructing Method," Power Electronics, IEEE Transactions on , vol.27, no.2, pp.905-915, Feb. 2012

[14] H.A. Toliyat, M. Wlas and Z. Krzemiriski, "Neural-NetworkBased Parameter Estimations of Induction Motors," Industrial Electronics, IEEE Transactions on , vol.55, no.4, pp.1783-1794, April 2008

[15] M. Rashed, P.F.A. MacConnell and A.F. Stronach, "Nonlinear adaptive state-feedback speed control of a voltage-fed induction motor with varying parameters," Industry Applications, IEEE Transactions on , vol.42, no.3, pp. 723- 732, May-June 2006

[16] E.V.N Souza and S.R. Naidu,; , "Simulation of the Three Phase Induction Machine Including the Effects of Saturation," IEEE Industrial Electronics, IECON 2006 - 32nd Annual Conference on , vol., no., pp.896-901, 6-10 Nov. 2006

[17] B. Shen, B. Mwinyiwiwa, Y. Zhang and B. Ooi; , "Sensorless Maximum Power Point Tracking of Wind by DFIG Using Rotor Position Phase Lock Loop (PLL)," Power Electronics, IEEE Transactions on , vol.24, no.4, pp.942-951, April 2009 